\title{
Transition Metal-Carbon Complexes. A Theoretical Study
}

\section{Andreas Krapp, Krishna K. Pandey and Gernot Frenking}

Supporting Information. Complete References 51 and 53. Table with coordinates and energies of the calculated compounds.

Reference 51: Gaussian03: Frisch, M.J.; Trucks, G.W.; Schlegel, H.B.; Scuseria, G.E.; Robb, M.A.; Cheeseman, J.R.; Montgomery, J.A., Jr; Vreven, T.; Kudin, K.N.; Burant, J.C.; Milliam, J.M; Iyengar, S.S.; Tomasi, J.; Barone, V.; Mennucci, B.; Cossi, M.; Scalmani, G.; Rega, N.; Petersson, G.A.; Nakatsuji, H.; Hada, M.; Ehara, M.; Toyota, K.; Fukuda, R; Hasegawa, J.; Ishida, M.; Nakajima, T;. Honda, Y.; Kitao, O.; Nakai, H.; Klene, M.; Li, X.; Knox, J. E.; Hratchian, H. P.; Cross, J. B.; Adamo, C.; Jaramillo, J.; Gomperts, R.; Stratmann, R. E.; Yazyev, O.; Austin, A. J. ; Cammi, R.; Pomelli, C.; Ochterski, J. W.; Ayala, P. Y.; Morokuma, K.; Voth, G. A.; Salvador, P.; Dannenberg, J. J.; Zakrzewski, V. G.; Dapprich, S. Daniels, A. D.; Strain, M. C.; Farkas, O.; Malick, D. K.; Rabuck, A. D.; Raghavachari, K; Foresman, J. B.; Ortiz, J. V.; Cui, Q. ; Baboul, A. G.; Clifford, S.; Cioslowski, J.; Stefanov, B. B.; Liu, G.; Liashenko, A.; Piskorz, P.; Komaromi, I; Martin, R. L.; Fox, D. J.; Keith, T.; Al-Laham, M. A.; Peng, C. Y.; Nanayakkara, A.; Challacombe, M.; Gill, P. M. W.; Johnson, B.; Chen, W.; Wong, C. Gonzalez, M. W.; Pople, J.A. Gaussian, Inc., Pittsburgh PA, 2003.

Reference 53: MOLPRO, a package of ab initio programs, version 2006.1: Werner, H.-J.; Knowles, P. J.; Lindh, R.; Manby, F.R. Schütz, M.; Celani, P.; Korona, T.; Rauhut, G.; Amos, R. D.; Bernhardsson, A.; Berning, A.; Cooper, D. L.; Deegan, M. J. O.; Dobbyn, A.J.; Eckert, F.; Hampel, C.; Hetzer, G.; Lloyd, A. W.; McNicholas, S. J.; Meyer, W.; Mura, M. E.; Nicklaß, A.; Palmieri, P.; Pitzer, R.; Schumann, U.; Stoll, H.; Stone, A. J.; Tarroni, R.; Thorsteinsson; T. 
Cartesian Coordinates ( $\AA$ ) of the optimized structures and total energies (a.u.).

The total energies in ADF are always given with respect to the atomic fragments. We used the standard atomic fragments out of an "create"-run for the TZ2P (all atoms except Fe) and TZ2P+ $(\mathrm{Fe})$ basissets and a small frozen core. The ZORA approximation was applied.

\begin{tabular}{|c|c|c|c|}
\hline \multicolumn{4}{|c|}{$E(\mathrm{a} . \mathrm{u})=-5.58357}$. \\
\hline $\mathrm{C}$ & 0.000000 & 0.000000 & -1.633912 \\
\hline $\mathrm{Fe}$ & 0.000000 & 0.000000 & -0.086116 \\
\hline $\mathrm{P}$ & 0.000000 & 2.261779 & 0.118063 \\
\hline $\mathrm{C}$ & 0.000000 & 2.817021 & 1.870375 \\
\hline $\mathrm{P}$ & 0.000000 & -2.261779 & 0.118063 \\
\hline $\mathrm{C}$ & 0.000000 & -2.817021 & 1.870375 \\
\hline $\mathrm{Cl}$ & 2.087789 & 0.000000 & 0.757587 \\
\hline $\mathrm{Cl}$ & -2.087789 & 0.000000 & 0.757587 \\
\hline $\mathrm{C}$ & -1.442226 & -3.126321 & -0.622587 \\
\hline $\mathrm{C}$ & 1.442226 & -3.126321 & -0.622587 \\
\hline $\mathrm{C}$ & -1.442226 & 3.126321 & -0.622587 \\
\hline $\mathrm{C}$ & 1.442226 & 3.126321 & -0.622587 \\
\hline $\mathrm{H}$ & 0.000000 & 3.914711 & 1.930465 \\
\hline $\mathrm{H}$ & -0.894141 & 2.417572 & 2.366473 \\
\hline $\mathrm{H}$ & 0.894141 & 2.417572 & 2.366473 \\
\hline $\mathrm{H}$ & -1.387530 & 4.206996 & -0.430074 \\
\hline $\mathrm{H}$ & -1.449597 & 2.946090 & -1.705902 \\
\hline $\mathrm{H}$ & -2.362817 & 2.712536 & -0.192453 \\
\hline $\mathrm{H}$ & 1.387530 & 4.206996 & -0.430074 \\
\hline $\mathrm{H}$ & 2.362817 & 2.712536 & -0.192453 \\
\hline $\mathrm{H}$ & 1.449597 & 2.946090 & -1.705902 \\
\hline $\mathrm{H}$ & -1.387530 & -4.206996 & -0.430074 \\
\hline $\mathrm{H}$ & -2.362817 & -2.712536 & -0.192453 \\
\hline $\mathrm{H}$ & -1.449597 & -2.946090 & -1.705902 \\
\hline $\mathrm{H}$ & 1.387530 & -4.206996 & -0.430074 \\
\hline $\mathrm{H}$ & 1.449597 & -2.946090 & -1.705902 \\
\hline $\mathrm{H}$ & 2.362817 & -2.712536 & -0.192453 \\
\hline $\mathrm{H}$ & 0.000000 & -3.914711 & 1.930465 \\
\hline $\mathrm{H}$ & 0.894141 & -2.417572 & 2.366473 \\
\hline $\mathrm{H}$ & & 572 & \\
\hline
\end{tabular}

$1 \mathrm{Ru}$ :

\begin{tabular}{lrrr}
$\mathrm{E}(\mathrm{a} . \mathrm{u})=-5.59963$. & & \\
$\mathrm{C}$ & 0.000000 & 0.000000 & -1.767720 \\
$\mathrm{RU}$ & 0.000000 & 0.000000 & -0.107151 \\
$\mathrm{P}$ & 0.000000 & 2.378664 & 0.164019 \\
& & & \multicolumn{1}{l}{$\mathrm{S} 2$} \\
$\mathrm{C}$ & 0.000000 & 2.895592 & 1.927976 \\
$\mathrm{P}$ & 0.000000 & -2.378664 & 0.164019 \\
$\mathrm{C}$ & 0.000000 & -2.895592 & 1.927976 \\
$\mathrm{Cl}$ & 2.234642 & 0.000000 & 0.739033 \\
$\mathrm{C} 1$ & -2.234642 & 0.000000 & 0.739033 \\
$\mathrm{C}$ & -1.452870 & -3.245462 & -0.550396 \\
$\mathrm{C}$ & 1.452870 & -3.245462 & -0.550396 \\
$\mathrm{C}$ & -1.452870 & 3.245462 & -0.550396 \\
$\mathrm{C}$ & 1.452870 & 3.245462 & -0.550396
\end{tabular}




\begin{tabular}{|c|c|c|c|}
\hline $\mathrm{H}$ & 0.000000 & 3.991757 & 2.012364 \\
\hline $\mathrm{H}$ & -0.894256 & 2.487284 & 2.417871 \\
\hline $\mathrm{H}$ & 0.894256 & 2.487284 & 2.417871 \\
\hline $\mathrm{H}$ & -1.422362 & 4.320495 & -0.324310 \\
\hline $\mathrm{H}$ & -1.459755 & 3.096433 & -1.638304 \\
\hline $\mathrm{H}$ & -2.363486 & 2.796716 & -0.132942 \\
\hline $\mathrm{H}$ & 1.422362 & 4.320495 & -0.324310 \\
\hline $\mathrm{H}$ & 2.363486 & 2.796716 & -0.132942 \\
\hline $\mathrm{H}$ & 1.459755 & 3.096433 & -1.638304 \\
\hline $\mathrm{H}$ & -1.422362 & -4.320495 & -0.324310 \\
\hline $\mathrm{H}$ & -2.363486 & -2.796716 & -0.132942 \\
\hline $\mathrm{H}$ & -1.459755 & -3.096433 & -1.638304 \\
\hline $\mathrm{H}$ & 1.422362 & -4.320495 & -0.324310 \\
\hline $\mathrm{H}$ & 1.459755 & -3.096433 & -1.638304 \\
\hline $\mathrm{H}$ & 2.363486 & -2.796716 & -0.132942 \\
\hline $\mathrm{H}$ & 0.000000 & -3.991757 & 2.012364 \\
\hline $\mathrm{H}$ & 0.894256 & -2.487284 & 2.417871 \\
\hline $\mathrm{H}$ & -0.894256 & -2.487284 & 2.417871 \\
\hline
\end{tabular}

\section{$2 \mathrm{Fe}$ :}

\begin{tabular}{|c|c|c|c|}
\hline \multicolumn{4}{|c|}{$E($ a.u. $)=-6.48719$} \\
\hline C & 0.000000 & 0.000000 & -1.46497 \\
\hline $\mathrm{Fe}$ & 0.000000 & 0.000000 & 0.17731 \\
\hline $\mathrm{P}$ & 0.000000 & -2.224992 & -0.029785 \\
\hline $\mathrm{C}$ & 1.508980 & 0.000000 & 1.178032 \\
\hline & 2.602847 & 0.000000 & 1.59390 \\
\hline C & -1.508980 & 0.000000 & 1.17803 \\
\hline & -2.602847 & 0.000000 & 1.59390 \\
\hline & 0.000000 & 2.224992 & -0.02978 \\
\hline C & -1.434110 & 2.947111 & -0.92757 \\
\hline$c$ & 1.434110 & 2.947111 & -0.92757 \\
\hline & 0.000000 & 3.158871 & 1.56009 \\
\hline & 0.000000 & 4.243611 & 1.38179 \\
\hline & -0.889390 & 2.882659 & 2.14137 \\
\hline & 0.889390 & 2.882659 & 2.14137 \\
\hline & -1.353276 & 4.041428 & -0.99234 \\
\hline & & & S3 \\
\hline & -1.465231 & 2.515437 & -1.93706 \\
\hline & -2.362976 & 2.677613 & -0.40738 \\
\hline & 1.353276 & 4.041428 & -0.99234 \\
\hline & 2.362976 & 2.677613 & -0.40738 \\
\hline & 1.465231 & 2.515437 & -1.93706 \\
\hline & -1.434110 & -2.947111 & -0.92757 \\
\hline & 1.434110 & -2.947111 & -0.92757 \\
\hline & 0.000000 & -3.158871 & 1.56009 \\
\hline & -1.353276 & -4.041428 & -0.99234 \\
\hline & -2.362976 & -2.677613 & $-0.4073 \varepsilon$ \\
\hline & -1.465231 & -2.515437 & -1.93706 \\
\hline & 1.353276 & -4.041428 & -0.99234 \\
\hline & 1.465231 & -2.515437 & -1.93706 \\
\hline & 2.362976 & -2.677613 & -0.40738 \\
\hline & 0.000000 & -4.243611 & 1.38179 \\
\hline & 0.889390 & -2.882659 & 2.14137 \\
\hline & -0.889390 & -2.882659 & $2.1413^{\prime}$ \\
\hline
\end{tabular}




\section{$2 \mathrm{Ru}$ :}

$\begin{array}{lrrr}\mathrm{E}(\mathrm{a} . \mathrm{u} .)=-6.47526 & & \\ \mathrm{C} & 0.000000 & 0.000000 & -1.625985 \\ \mathrm{Ru} & 0.000000 & 0.000000 & 0.122511 \\ \mathrm{P} & 0.000000 & -2.400575 & -0.009983 \\ \mathrm{C} & 1.685351 & 0.000000 & 1.184811 \\ \mathrm{O} & 2.835265 & 0.000000 & 1.398807 \\ \mathrm{C} & -1.685351 & 0.000000 & 1.184811 \\ \mathrm{O} & -2.835265 & 0.000000 & 1.398807 \\ \mathrm{P} & 0.000000 & 2.400575 & -0.009983 \\ \mathrm{C} & -1.434323 & 3.151509 & -0.884800 \\ \mathrm{C} & 1.434323 & 3.151509 & -0.884800 \\ \mathrm{C} & 0.000000 & 3.274244 & 1.611996 \\ \mathrm{H} & 0.000000 & 4.365288 & 1.478009 \\ \mathrm{H} & -0.888703 & 2.974143 & 2.182876 \\ \mathrm{H} & 0.888703 & 2.974143 & 2.182876 \\ \mathrm{H} & -1.354927 & 4.247561 & -0.916021 \\ \mathrm{H} & -1.466272 & 2.751986 & -1.907560 \\ \mathrm{H} & -2.363536 & 2.864563 & -0.374812 \\ \mathrm{H} & 1.354927 & 4.247561 & -0.916021 \\ \mathrm{H} & 2.363536 & 2.864563 & -0.374812 \\ \mathrm{H} & 1.466272 & 2.751986 & -1.907560 \\ \mathrm{C} & -1.434323 & -3.151509 & -0.884800 \\ \mathrm{C} & 1.434323 & -3.151509 & -0.884800 \\ \mathrm{C} & 0.000000 & -3.274244 & 1.611996 \\ \mathrm{H} & -1.354927 & -4.247561 & -0.916021 \\ \mathrm{H} & -2.363536 & -2.864563 & -0.374812 \\ & & & \\ & & & \mathrm{~S} 4 \\ \mathrm{H} & -1.466272 & -2.751986 & -1.907560 \\ \mathrm{H} & 1.354927 & -4.247561 & -0.916021 \\ \mathrm{H} & 1.466272 & -2.751986 & -1.907560 \\ \mathrm{H} & 2.363536 & -2.864563 & -0.374812 \\ \mathrm{H} & 0.000000 & -4.365288 & 1.478009 \\ \mathrm{H} & 0.888703 & -2.974143 & 2.182876 \\ \mathrm{H} & -0.888703 & -2.974143 & 2.182876 \\ ---------------------10-----------\end{array}$

3Fe_ax:

$\begin{array}{lrrr}\mathrm{E}(\mathrm{a} . \mathrm{u} .)=-2.81215 & & \\ \mathrm{Fe} & 0.000000 & 0.000000 & 0.047529 \\ \mathrm{C} & -1.816392 & 0.000000 & 0.049502 \\ \mathrm{C} & 0.908196 & -1.573042 & 0.049502 \\ \mathrm{C} & 0.908196 & 1.573042 & 0.049502 \\ \mathrm{C} & 0.000000 & 0.000000 & 2.041378 \\ \mathrm{C} & 0.000000 & 0.000000 & -1.593143 \\ \mathrm{O} & -2.965831 & 0.000000 & -0.002576 \\ \mathrm{O} & 1.482915 & 2.568485 & -0.002576 \\ \mathrm{O} & 1.482915 & -2.568485 & -0.002576 \\ \mathrm{O} & 0.000000 & 0.000000 & 3.185018 \\ - & & & \end{array}$

\section{Fe_eq :}

$E($ a.u. $)=-2.80156$

$\begin{array}{llll}\mathrm{Fe} & 0.000000 & 0.000000 & 0.095123 \\ \mathrm{C} & 1.491202 & 0.000000 & 1.182923\end{array}$




\begin{tabular}{|c|c|c|c|}
\hline C & 0.000000 & 0.000000 & -1.579740 \\
\hline $\mathrm{C}$ & -1.491202 & 0.000000 & 1.182923 \\
\hline C & 0.000000 & 1.818575 & -0.090547 \\
\hline C & 0.000000 & -1.818575 & -0.090547 \\
\hline 0 & 2.525193 & 0.000000 & 1.689460 \\
\hline 0 & -2.525193 & 0.000000 & 1.689460 \\
\hline O & 0.000000 & 2.963299 & -0.187136 \\
\hline 0 & 0.000000 & -2.963299 & -0.187136 \\
\hline
\end{tabular}

\section{Ru_ax :}

$\begin{array}{lrrr}\mathrm{E}(\mathrm{a} . \mathrm{u} .)=-2.79178 & & \\ \mathrm{Ru} & 0.000000 & 0.000000 & -0.090018 \\ \mathrm{C} & -1.976687 & 0.000000 & 0.089639 \\ \mathrm{C} & 0.988344 & -1.711861 & 0.089639 \\ \mathrm{C} & 0.988344 & 1.711861 & 0.089639 \\ \mathrm{C} & 0.000000 & 0.000000 & 2.387140\end{array}$

\begin{tabular}{|c|c|c|c|}
\hline & & & S5 \\
\hline C & 0.000000 & 0.000000 & -1.795981 \\
\hline & -3.126876 & 0.000000 & 0.06507 \\
\hline & 1.563438 & 2.707954 & 0.06507 \\
\hline & 1.563438 & -2.707954 & 0.065076 \\
\hline & 0.000000 & 0.000000 & 3.524219 \\
\hline
\end{tabular}

\section{Ru_eq :}

$\begin{array}{lrrr}\mathrm{E}(\mathrm{a} . \mathrm{u} .)=-2.78358 & & \\ \mathrm{Ru} & 0.000000 & 0.000000 & 0.054431 \\ \mathrm{C} & 1.658246 & 0.000000 & 1.211746 \\ \mathrm{C} & 0.000000 & 0.000000 & -1.729354 \\ \mathrm{C} & -1.658246 & 0.000000 & 1.211746 \\ \mathrm{C} & 0.000000 & 1.993145 & -0.063987 \\ \mathrm{C} & 0.000000 & -1.993145 & -0.063987 \\ \mathrm{O} & 2.758191 & 0.000000 & 1.555466 \\ \mathrm{O} & -2.758191 & 0.000000 & 1.555466 \\ \mathrm{O} & 0.000000 & 3.139733 & -0.045636 \\ \mathrm{O} & 0.000000 & -3.139733 & -0.045636 \\ - & & \end{array}$

\section{$4 \mathrm{Fe}:$}

$\begin{array}{lrrr} & \\ \mathrm{E}(\mathrm{a} . \mathrm{u} .)=-5.92096 & & \\ \mathrm{C} & 0.000000 & 2.844357 & 1.596961 \\ \mathrm{P} & 0.000000 & 2.261582 & -0.151261 \\ \mathrm{C} & 1.438707 & 3.137627 & -0.891265 \\ \mathrm{Fe} & 0.000000 & 0.000000 & -0.266017 \\ \mathrm{Cl} & -2.133166 & 0.000000 & 0.408691 \\ \mathrm{C} & 0.000000 & 0.000000 & -1.951506 \\ \mathrm{O} & 0.000000 & 0.000000 & -3.124895 \\ \mathrm{P} & 0.000000 & -2.261582 & -0.151261 \\ \mathrm{C} & 1.438707 & -3.137627 & -0.891265 \\ \mathrm{Cl} & 2.133166 & 0.000000 & 0.408691 \\ \mathrm{C} & -1.438707 & 3.137627 & -0.891265 \\ \mathrm{C} & 0.000000 & -2.844357 & 1.596961 \\ \mathrm{C} & -1.438707 & -3.137627 & -0.891265 \\ \mathrm{H} & 0.000000 & 3.942829 & 1.642059\end{array}$




\begin{tabular}{lrrr}
$\mathrm{H}$ & -0.894063 & 2.454185 & 2.100923 \\
$\mathrm{H}$ & 0.894063 & 2.454185 & 2.100923 \\
$\mathrm{H}$ & -1.379449 & 4.216512 & -0.689978 \\
$\mathrm{H}$ & -1.448118 & 2.971727 & -1.976999 \\
$\mathrm{H}$ & -2.362855 & 2.725854 & -0.466729 \\
$\mathrm{H}$ & 1.379449 & 4.216512 & -0.689978 \\
$\mathrm{H}$ & 2.362855 & 2.725854 & -0.466729 \\
$\mathrm{H}$ & 1.448118 & 2.971727 & -1.976999 \\
& & & \multicolumn{1}{l}{$\mathrm{S} 6$} \\
$\mathrm{H}$ & -1.379449 & -4.216512 & -0.689978 \\
$\mathrm{H}$ & -2.362855 & -2.725854 & -0.466729 \\
$\mathrm{H}$ & -1.448118 & -2.971727 & -1.976999 \\
$\mathrm{H}$ & 1.379449 & -4.216512 & -0.689978 \\
$\mathrm{H}$ & 1.448118 & -2.971727 & -1.976999 \\
$\mathrm{H}$ & 2.362855 & -2.725854 & -0.466729 \\
$\mathrm{H}$ & 0.000000 & -3.942829 & 1.642059 \\
$\mathrm{H}$ & 0.894063 & -2.454185 & 2.100923 \\
$\mathrm{H}$ & -0.894063 & -2.454185 & 2.100923 \\
--------------------------1
\end{tabular}

\section{Ru :}

\begin{tabular}{|c|c|c|c|}
\hline \multicolumn{4}{|c|}{$E($ a.u. $)=-5.92914$} \\
\hline $\mathrm{C}$ & 0.000000 & 2.935858 & 1.644891 \\
\hline $\mathrm{P}$ & 0.000000 & 2.370455 & -0.111155 \\
\hline $\mathrm{C}$ & 1.448722 & 3.243525 & -0.832978 \\
\hline $\mathrm{Ru}$ & 0.000000 & 0.000000 & -0.251251 \\
\hline $\mathrm{Cl}$ & -2.303394 & 0.000000 & 0.331634 \\
\hline $\mathrm{C}$ & 0.000000 & 0.000000 & -2.034489 \\
\hline 0 & 0.000000 & 0.000000 & -3.210500 \\
\hline $\mathrm{P}$ & 0.000000 & -2.370455 & -0.111155 \\
\hline C & 1.448722 & -3.243525 & -0.832978 \\
\hline $\mathrm{Cl}$ & 2.303394 & 0.000000 & 0.331634 \\
\hline C & -1.448722 & 3.243525 & -0.832978 \\
\hline C & 0.000000 & -2.935858 & 1.644891 \\
\hline $\mathrm{C}$ & -1.448722 & -3.243525 & -0.832978 \\
\hline $\mathrm{H}$ & 0.000000 & 4.033958 & 1.701854 \\
\hline $\mathrm{H}$ & -0.894186 & 2.544199 & 2.148663 \\
\hline $\mathrm{H}$ & 0.894186 & 2.544199 & 2.148663 \\
\hline $\mathrm{H}$ & -1.408920 & 4.319751 & -0.614194 \\
\hline $\mathrm{H}$ & -1.459759 & 3.092141 & -1.920709 \\
\hline $\mathrm{H}$ & -2.363738 & 2.805297 & -0.413703 \\
\hline $\mathrm{H}$ & 1.408920 & 4.319751 & -0.614194 \\
\hline $\mathrm{H}$ & 2.363738 & 2.805297 & -0.413703 \\
\hline $\mathrm{H}$ & 1.459759 & 3.092141 & -1.920709 \\
\hline $\mathrm{H}$ & -1.408920 & -4.319751 & -0.614194 \\
\hline $\mathrm{H}$ & -2.363738 & -2.805297 & -0.413703 \\
\hline $\mathrm{H}$ & -1.459759 & -3.092141 & -1.920709 \\
\hline $\mathrm{H}$ & 1.408920 & -4.319751 & -0.614194 \\
\hline $\mathrm{H}$ & 1.459759 & -3.092141 & -1.920709 \\
\hline $\mathrm{H}$ & 2.363738 & -2.805297 & -0.413703 \\
\hline $\mathrm{H}$ & 0.000000 & -4.033958 & 1.701854 \\
\hline $\mathrm{H}$ & 0.894186 & -2.544199 & 2.148663 \\
\hline $\mathrm{H}$ & -0.894186 & -2.544199 & 2.148663 \\
\hline
\end{tabular}




\section{$5 \mathrm{Fe}$ :}

\begin{tabular}{|c|c|c|c|}
\hline \multicolumn{4}{|c|}{$E(\mathrm{a} \cdot \mathrm{u})=-6.88277}$. \\
\hline $\mathrm{C}$ & 0.000000 & 0.000000 & 1.768735 \\
\hline $\mathrm{Fe}$ & 0.000000 & 0.000000 & 0.001363 \\
\hline $\mathrm{P}$ & 0.000000 & 2.211725 & -0.000592 \\
\hline C & 0.000000 & 3.018299 & -1.657756 \\
\hline $\mathrm{P}$ & 0.000000 & -2.211725 & -0.000592 \\
\hline $\mathrm{C}$ & 0.000000 & -3.018299 & -1.657756 \\
\hline $\mathrm{C}$ & -1.531157 & 0.000000 & -0.881119 \\
\hline $\mathrm{C}$ & 1.531157 & 0.000000 & -0.881119 \\
\hline C & 1.434116 & -3.022075 & 0.826166 \\
\hline $\mathrm{C}$ & -1.434116 & -3.022075 & 0.826166 \\
\hline $\mathrm{C}$ & 1.434116 & 3.022075 & 0.826166 \\
\hline $\mathrm{C}$ & -1.434116 & 3.022075 & 0.826166 \\
\hline $\mathrm{H}$ & 0.000000 & 4.113628 & -1.562754 \\
\hline $\mathrm{H}$ & 0.890088 & 2.699072 & -2.215814 \\
\hline $\mathrm{H}$ & -0.890088 & 2.699072 & -2.215814 \\
\hline $\mathrm{H}$ & 1.350210 & 4.117163 & 0.775924 \\
\hline $\mathrm{H}$ & 1.472798 & 2.705556 & 1.876830 \\
\hline $\mathrm{H}$ & 2.362937 & 2.703062 & 0.335136 \\
\hline $\mathrm{H}$ & -1.350210 & 4.117163 & 0.775924 \\
\hline $\mathrm{H}$ & -2.362937 & 2.703062 & 0.335136 \\
\hline $\mathrm{H}$ & -1.472798 & 2.705556 & 1.876830 \\
\hline $\mathrm{H}$ & 1.350210 & -4.117163 & 0.775924 \\
\hline $\mathrm{H}$ & 2.362937 & -2.703062 & 0.335136 \\
\hline $\mathrm{H}$ & 1.472798 & -2.705556 & 1.876830 \\
\hline $\mathrm{H}$ & -1.350210 & -4.117163 & 0.775924 \\
\hline $\mathrm{H}$ & -1.472798 & -2.705556 & 1.876830 \\
\hline $\mathrm{H}$ & -2.362937 & -2.703062 & 0.335136 \\
\hline $\mathrm{H}$ & 0.000000 & -4.113628 & -1.562754 \\
\hline $\mathrm{H}$ & -0.890088 & -2.699072 & -2.215814 \\
\hline $\mathrm{H}$ & 0.890088 & -2.699072 & -2.215814 \\
\hline 0 & 0.000000 & 0.000000 & 2.941880 \\
\hline 0 & -2.547725 & 0.000000 & -1.466742 \\
\hline ? & 2.547725 & 0.000000 & -1.466742 \\
\hline
\end{tabular}

$5 \mathrm{Ru}$ :

\begin{tabular}{lrrr}
$\mathrm{E}(\mathrm{a} . \mathrm{u})=-6.87071$. & & \\
$\mathrm{C}$ & 0.000000 & 0.000000 & 1.922318 \\
$\mathrm{Ru}$ & 0.000000 & 0.000000 & 0.001398 \\
$\mathrm{P}$ & 0.000000 & 2.359400 & -0.000383 \\
$\mathrm{C}$ & 0.000000 & 3.164009 & -1.659064 \\
$\mathrm{P}$ & 0.000000 & -2.359400 & -0.000383 \\
$\mathrm{C}$ & 0.000000 & -3.164009 & -1.659064 \\
& & & \multicolumn{1}{l}{} \\
$\mathrm{C}$ & & & \multicolumn{1}{l}{$\mathrm{S} 8$} \\
$\mathrm{C}$ & -1.663404 & 0.000000 & -0.958735 \\
$\mathrm{C}$ & 1.663404 & 0.000000 & -0.958735 \\
$\mathrm{C}$ & 1.435642 & -3.167234 & 0.827321 \\
$\mathrm{C}$ & -1.435642 & -3.167234 & 0.827321 \\
$\mathrm{C}$ & 1.435642 & 3.167234 & 0.827321 \\
$\mathrm{C}$ & -1.435642 & 3.167234 & 0.827321 \\
$\mathrm{H}$ & 0.000000 & 4.259839 & -1.568122 \\
$\mathrm{H}$ & 0.889449 & 2.841666 & -2.216554 \\
$\mathrm{H}$ & -0.889449 & 2.841666 & -2.216554 \\
$\mathrm{H}$ & 1.354702 & 4.262865 & 0.781028
\end{tabular}




\begin{tabular}{|c|c|c|c|}
\hline $\mathrm{H}$ & 1.475267 & 2.845821 & 1.876583 \\
\hline $\mathrm{H}$ & 2.363395 & 2.846364 & 0.335211 \\
\hline $\mathrm{H}$ & -1.354702 & 4.262865 & 0.781028 \\
\hline $\mathrm{H}$ & -2.363395 & 2.846364 & 0.335211 \\
\hline $\mathrm{H}$ & -1.475267 & 2.845821 & 1.876583 \\
\hline $\mathrm{H}$ & 1.354702 & -4.262865 & 0.781028 \\
\hline $\mathrm{H}$ & 2.363395 & -2.846364 & 0.335211 \\
\hline $\mathrm{H}$ & 1.475267 & -2.845821 & 1.876583 \\
\hline $\mathrm{H}$ & -1.354702 & -4.262865 & 0.781028 \\
\hline $\mathrm{H}$ & -1.475267 & -2.845821 & 1.876583 \\
\hline $\mathrm{H}$ & -2.363395 & -2.846364 & 0.335211 \\
\hline $\mathrm{H}$ & 0.000000 & -4.259839 & -1.568122 \\
\hline $\mathrm{H}$ & -0.889449 & -2.841666 & -2.216554 \\
\hline $\mathrm{H}$ & 0.889449 & -2.841666 & -2.216554 \\
\hline 0 & 0.000000 & 0.000000 & 3.093888 \\
\hline 0 & -2.677912 & 0.000000 & -1.544749 \\
\hline 0 & 2.677912 & 0.000000 & -1.544749 \\
\hline
\end{tabular}

\section{Fe:}

$\begin{array}{lrrr}\mathrm{E}(\mathrm{a} . \mathrm{u} .)=-3.21117 & & \\ \mathrm{Fe} & 0.000000 & 0.000000 & 0.000000 \\ \mathrm{C} & 0.900364 & -1.559476 & 0.000000 \\ \mathrm{C} & 0.900364 & 1.559476 & 0.000000 \\ \mathrm{C} & -1.800728 & 0.000000 & 0.000000 \\ \mathrm{C} & 0.000000 & 0.000000 & -1.803041 \\ \mathrm{C} & 0.000000 & 0.000000 & 1.803041 \\ \mathrm{O} & 0.000000 & 0.000000 & -2.953817 \\ \mathrm{O} & 0.000000 & 0.000000 & 2.953817 \\ \mathrm{O} & 1.477183 & 2.558556 & 0.000000 \\ \mathrm{O} & -2.954366 & 0.000000 & 0.000000 \\ \mathrm{O} & 1.477183 & -2.558556 & 0.000000 \\ - & & \end{array}$

S9

$6 \mathrm{Ru}:$

$\begin{array}{lrrr}\mathrm{E}(\mathrm{a} . \mathrm{u} .)=-3.19391 & & \\ \mathrm{Ru} & 0.000000 & 0.000000 & 0.000000 \\ \mathrm{C} & 0.976662 & -1.691628 & 0.000000 \\ \mathrm{C} & 0.976662 & 1.691628 & 0.000000 \\ \mathrm{C} & -1.953324 & 0.000000 & 0.000000 \\ \mathrm{C} & 0.000000 & 0.000000 & -1.953293 \\ \mathrm{C} & 0.000000 & 0.000000 & 1.953293 \\ \mathrm{O} & 0.000000 & 0.000000 & -3.102677 \\ \mathrm{O} & 0.000000 & 0.000000 & 3.102677 \\ \mathrm{O} & 1.553670 & 2.691035 & 0.000000 \\ \mathrm{O} & -3.107339 & 0.000000 & 0.000000 \\ \mathrm{O} & 1.553670 & -2.691035 & 0.000000\end{array}$

$7 \mathrm{Fe} S \mathrm{~S}$ :

$\begin{array}{llll}\mathrm{E}(\mathrm{a} . \mathrm{u} .)=-5.30812 & & \\ \mathrm{C} & -1.438004 & 1.962795 & -2.234742 \\ \mathrm{P} & 0.000000 & 1.652781 & -1.116990\end{array}$ 


\begin{tabular}{|c|c|c|c|}
\hline C & 0.000000 & 3.174366 & -0.060612 \\
\hline $\mathrm{Fe}$ & 0.000000 & 0.000000 & 0.191224 \\
\hline $\mathrm{Cl}$ & 2.085732 & 0.000000 & 0.846556 \\
\hline $\mathrm{Cl}$ & -2.085732 & 0.000000 & 0.846556 \\
\hline $\mathrm{P}$ & 0.000000 & -1.652781 & -1.116990 \\
\hline $\mathrm{C}$ & 1.438004 & -1.962795 & -2.234742 \\
\hline $\mathrm{C}$ & 1.438004 & 1.962795 & -2.234742 \\
\hline $\mathrm{C}$ & -1.438004 & -1.962795 & -2.234742 \\
\hline C & 0.000000 & -3.174366 & -0.060612 \\
\hline $\mathrm{H}$ & 1.358684 & 2.959423 & -2.692899 \\
\hline $\mathrm{H}$ & 2.362731 & 1.892814 & -1.648006 \\
\hline $\mathrm{H}$ & 1.470951 & 1.207726 & -3.031209 \\
\hline $\mathrm{H}$ & 0.000000 & 4.083062 & -0.680443 \\
\hline $\mathrm{H}$ & -0.892583 & 3.165687 & 0.578596 \\
\hline $\mathrm{H}$ & 0.892583 & 3.165687 & 0.578596 \\
\hline $\mathrm{H}$ & 1.358684 & -2.959423 & -2.692899 \\
\hline $\mathrm{H}$ & 1.470951 & -1.207726 & -3.031209 \\
\hline $\mathrm{H}$ & 2.362731 & -1.892814 & -1.648006 \\
\hline $\mathrm{H}$ & -1.358684 & -2.959423 & -2.692899 \\
\hline $\mathrm{H}$ & -2.362731 & -1.892814 & -1.648006 \\
\hline $\mathrm{H}$ & -1.470951 & -1.207726 & -3.031209 \\
\hline $\mathrm{H}$ & 0.000000 & -4.083062 & -0.680443 \\
\hline $\mathrm{H}$ & 0.892583 & -3.165687 & 0.578596 \\
\hline $\mathrm{H}$ & -0.892583 & -3.165687 & 0.578596 \\
\hline $\mathrm{H}$ & -1.358684 & 2.959423 & -2.692899 \\
\hline $\mathrm{H}$ & -1.470951 & 1.207726 & -3.031209 \\
\hline $\mathrm{H}$ & -2.362731 & 1.892814 & -1.648006 \\
\hline
\end{tabular}

\section{S10}

7Fe_T:

$\begin{array}{lrrr}\mathrm{E}(\mathrm{a} . \mathrm{u} .)=-5.31539 & & \\ \mathrm{C} & 0.000000 & 2.976335 & 1.901534 \\ \mathrm{P} & 0.000000 & 2.276468 & 0.194498 \\ \mathrm{C} & 1.431451 & 3.137270 & -0.589981 \\ \mathrm{Fe} & 0.000000 & 0.000000 & 0.191700 \\ \mathrm{Cl} & -2.201356 & 0.000000 & 0.533300 \\ \mathrm{P} & 0.000000 & -2.276468 & 0.194498 \\ \mathrm{C} & 1.431451 & -3.137270 & -0.589981 \\ \mathrm{Cl} & 2.201356 & 0.000000 & 0.533300 \\ \mathrm{C} & -1.431451 & 3.137270 & -0.589981 \\ \mathrm{C} & 0.000000 & -2.976335 & 1.901534 \\ \mathrm{C} & -1.431451 & -3.137270 & -0.589981 \\ \mathrm{H} & 0.000000 & 4.075586 & 1.879942 \\ \mathrm{H} & -0.893052 & 2.620401 & 2.432264 \\ \mathrm{H} & 0.893052 & 2.620401 & 2.432264 \\ \mathrm{H} & -1.350159 & 4.225519 & -0.458950 \\ \mathrm{H} & -1.453358 & 2.903924 & -1.663251 \\ \mathrm{H} & -2.361898 & 2.770124 & -0.140196 \\ \mathrm{H} & 1.350159 & 4.225519 & -0.458950 \\ \mathrm{H} & 2.361898 & 2.770124 & -0.140196 \\ \mathrm{H} & 1.453358 & 2.903924 & -1.663251 \\ \mathrm{H} & -1.350159 & -4.225519 & -0.458950 \\ \mathrm{H} & -2.361898 & -2.770124 & -0.140196 \\ \mathrm{H} & -1.453358 & -2.903924 & -1.663251 \\ \mathrm{H} & 1.350159 & -4.225519 & -0.458950 \\ \mathrm{H} & 1.453358 & -2.903924 & -1.663251 \\ \mathrm{H} & 2.361898 & -2.770124 & -0.140196 \\ \mathrm{H} & 0.000000 & -4.075586 & 1.879942\end{array}$




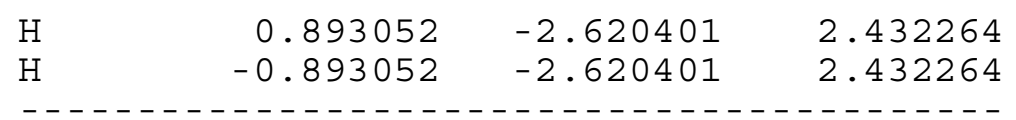

\section{$7 \mathrm{Ru}$ S :}

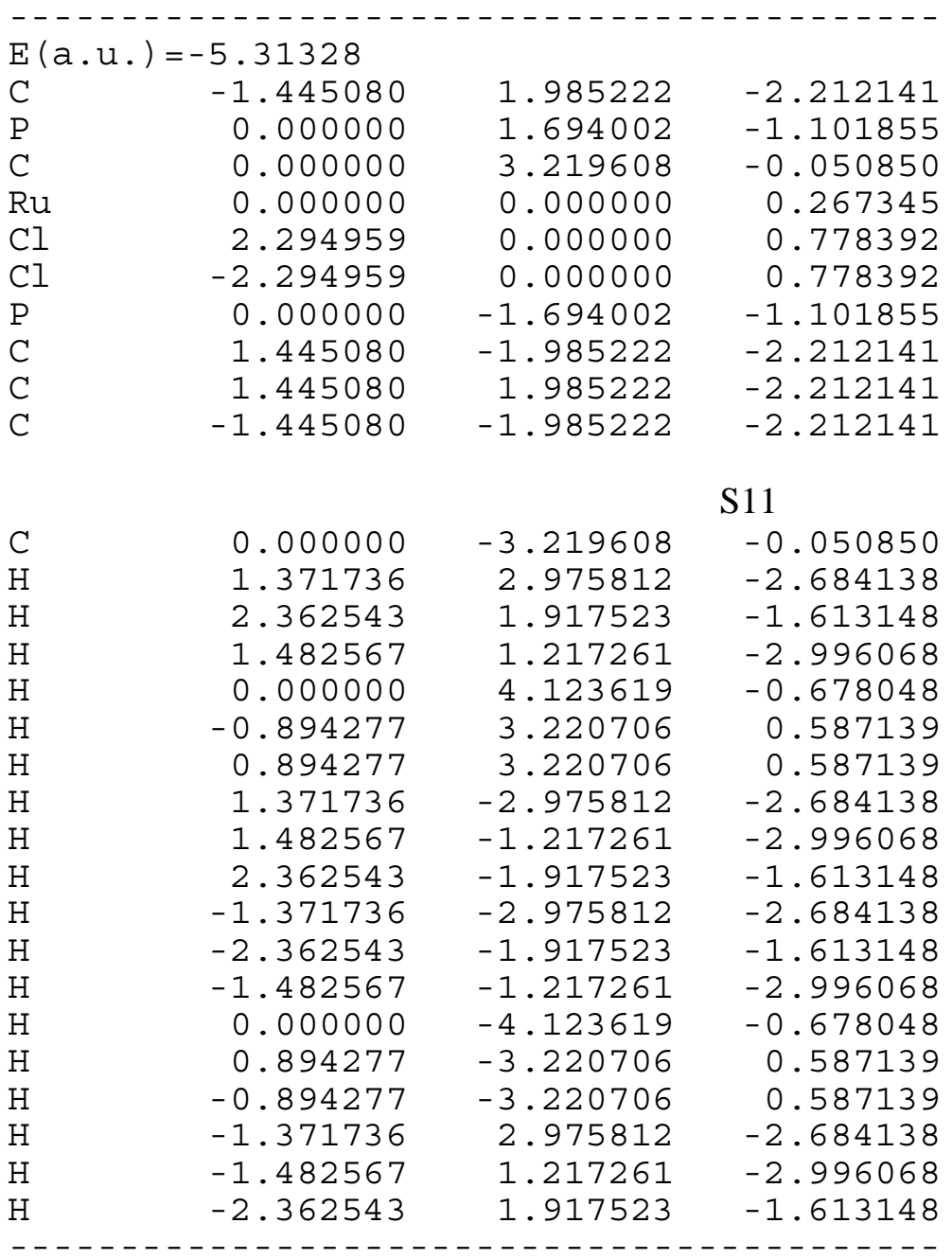

7Ru_T:

$\begin{array}{lrrr}\mathrm{E}(\mathrm{a} . \mathrm{u} .)=-5.28937 & & \\ \mathrm{C} & 0.000000 & 3.133057 & 1.889688 \\ \mathrm{P} & 0.000000 & 2.367896 & 0.207870 \\ \mathrm{C} & 1.437045 & 3.200694 & -0.596927 \\ \mathrm{Ru} & 0.000000 & 0.000000 & 0.203947 \\ \mathrm{C} 1 & -2.325377 & 0.000000 & 0.488536 \\ \mathrm{P} & 0.000000 & -2.367896 & 0.207870 \\ \mathrm{C} & 1.437045 & -3.200694 & -0.596927 \\ \mathrm{Cl} & 2.325377 & 0.000000 & 0.488536 \\ \mathrm{C} & -1.437045 & 3.200694 & -0.596927 \\ \mathrm{C} & 0.000000 & -3.133057 & 1.889688 \\ \mathrm{C} & -1.437045 & -3.200694 & -0.596927 \\ \mathrm{H} & 0.000000 & 4.230875 & 1.825545 \\ \mathrm{H} & -0.892426 & 2.800295 & 2.436926 \\ \mathrm{H} & 0.892426 & 2.800295 & 2.436926 \\ \mathrm{H} & -1.371874 & 4.292480 & -0.487722 \\ \mathrm{H} & -1.455517 & 2.943688 & -1.664857\end{array}$




\begin{tabular}{|c|c|c|c|}
\hline $\mathrm{H}$ & -2.364258 & 2.829864 & -0.142078 \\
\hline $\mathrm{H}$ & 1.371874 & 4.292480 & -0.487722 \\
\hline $\mathrm{H}$ & 2.364258 & 2.829864 & -0.142078 \\
\hline $\mathrm{H}$ & 1.455517 & 2.943688 & -1.664857 \\
\hline $\mathrm{H}$ & -1.371874 & -4.292480 & -0.487722 \\
\hline $\mathrm{H}$ & -2.364258 & -2.829864 & -0.142078 \\
\hline \multirow[t]{2}{*}{$\mathrm{H}$} & -1.455517 & -2.943688 & -1.664857 \\
\hline & & \multicolumn{2}{|c|}{$\mathrm{S} 12$} \\
\hline $\mathrm{H}$ & 1.371874 & -4.292480 & -0.487722 \\
\hline $\mathrm{H}$ & 1.455517 & -2.943688 & -1.664857 \\
\hline $\mathrm{H}$ & 2.364258 & -2.829864 & -0.142078 \\
\hline $\mathrm{H}$ & 0.000000 & -4.230875 & 1.825545 \\
\hline $\mathrm{H}$ & 0.892426 & -2.800295 & 2.436926 \\
\hline $\mathrm{H}$ & -0.892426 & -2.800295 & 2.436926 \\
\hline
\end{tabular}

\section{Fe_S:}

\begin{tabular}{|c|c|c|c|}
\hline \multicolumn{4}{|c|}{6.24990} \\
\hline $\mathrm{Fe}$ & 0.000000 & 0.000000 & 0.196394 \\
\hline $\mathrm{P}$ & 0.000000 & 2.196439 & -0.023130 \\
\hline $\mathrm{C}$ & 0.000000 & 3.146958 & 1.555096 \\
\hline D & 0.000000 & -2.196439 & -0.023130 \\
\hline C & 0.000000 & -3.146958 & 1.55509 \\
\hline C & 1.545123 & 0.000000 & 0.97875 \\
\hline 0 & 2.475813 & 0.000000 & 1.70799 \\
\hline C & -1.545123 & 0.000000 & 0.97875 \\
\hline & -2.475813 & 0.000000 & 1.70799 \\
\hline & -1.430695 & -2.950236 & $-0.9253 c$ \\
\hline & 1.430695 & -2.950236 & -0.92530 \\
\hline & -1.430695 & 2.950236 & $-0.9253 c$ \\
\hline$C$ & 1.430695 & 2.950236 & -0.92530 \\
\hline & 0.000000 & 4.229452 & 1.36334 \\
\hline & -0.889984 & 2.874420 & $2.1370 \varepsilon$ \\
\hline & 0.889984 & 2.874420 & 2.13708 \\
\hline & -1.330186 & 4.043834 & -0.98676 \\
\hline & -1.484542 & 2.538346 & -1.94242 \\
\hline & -2.361341 & 2.695325 & -0.40176 \\
\hline & 1.330186 & 4.043834 & -0.98676 \\
\hline & 2.361341 & 2.695325 & -0.40176 \\
\hline & 1.484542 & 2.538346 & -1.94242 \\
\hline & -1.330186 & -4.043834 & -0.986769 \\
\hline & -2.361341 & -2.695325 & -0.40176 \\
\hline & -1.484542 & -2.538346 & -1.94242 \\
\hline & 1.330186 & -4.043834 & -0.98676 \\
\hline & 1.484542 & -2.538346 & -1.94242 \\
\hline & 2.361341 & -2.695325 & -0.40176 \\
\hline & 0.000000 & -4.229452 & 1.363340 \\
\hline & 0.889984 & -2.874420 & 2.13708 \\
\hline & -0.889984 & -2.874420 & $2.1370 \varepsilon$ \\
\hline
\end{tabular}

\section{S13}

8Fe_T: 


\begin{tabular}{|c|c|c|c|}
\hline \multicolumn{4}{|c|}{$E($ a.u. $)=-6.24849$} \\
\hline $\mathrm{Fe}$ & 0.000000 & 0.000000 & 0.430568 \\
\hline $\mathrm{P}$ & 0.000000 & 2.145415 & -0.248509 \\
\hline $\mathrm{C}$ & 0.000000 & 3.395979 & 1.110913 \\
\hline $\mathrm{P}$ & 0.000000 & -2.145415 & -0.248509 \\
\hline $\mathrm{C}$ & 0.000000 & -3.395979 & 1.110913 \\
\hline $\mathrm{C}$ & 1.342275 & 0.000000 & 1.583360 \\
\hline 0 & 2.216926 & 0.000000 & 2.364546 \\
\hline $\mathrm{C}$ & -1.342275 & 0.000000 & 1.583360 \\
\hline 0 & -2.216926 & 0.000000 & 2.364546 \\
\hline $\mathrm{C}$ & -1.429473 & -2.736655 & -1.271513 \\
\hline $\mathrm{C}$ & 1.429473 & -2.736655 & -1.271513 \\
\hline $\mathrm{C}$ & -1.429473 & 2.736655 & -1.271513 \\
\hline $\mathrm{C}$ & 1.429473 & 2.736655 & -1.271513 \\
\hline $\mathrm{H}$ & 0.000000 & 4.420433 & 0.710717 \\
\hline $\mathrm{H}$ & -0.888833 & 3.246589 & 1.737769 \\
\hline $\mathrm{H}$ & 0.888833 & 3.246589 & 1.737769 \\
\hline $\mathrm{H}$ & -1.338259 & 3.808745 & -1.500855 \\
\hline $\mathrm{H}$ & -1.471441 & 2.169248 & -2.210905 \\
\hline $\mathrm{H}$ & -2.364106 & 2.562179 & -0.722274 \\
\hline $\mathrm{H}$ & 1.338259 & 3.808745 & -1.500855 \\
\hline $\mathrm{H}$ & 2.364106 & 2.562179 & -0.722274 \\
\hline $\mathrm{H}$ & 1.471441 & 2.169248 & -2.210905 \\
\hline $\mathrm{H}$ & -1.338259 & -3.808745 & -1.500855 \\
\hline $\mathrm{H}$ & -2.364106 & -2.562179 & -0.722274 \\
\hline $\mathrm{H}$ & -1.471441 & -2.169248 & -2.210905 \\
\hline $\mathrm{H}$ & 1.338259 & -3.808745 & -1.500855 \\
\hline $\mathrm{H}$ & 1.471441 & -2.169248 & -2.210905 \\
\hline $\mathrm{H}$ & 2.364106 & -2.562179 & -0.722274 \\
\hline $\mathrm{H}$ & 0.000000 & -4.420433 & 0.710717 \\
\hline $\mathrm{H}$ & 0.888833 & -3.246589 & 1.737769 \\
\hline $\mathrm{H}$ & -0.888833 & -3.246589 & 1.737769 \\
\hline
\end{tabular}

\section{Ru S :}

$\begin{array}{lrrr}\mathrm{E}(\mathrm{a} . \mathrm{u} .)=-6.26178 & & \\ \mathrm{Ru} & 0.000000 & 0.000000 & 0.186247 \\ \mathrm{P} & 0.000000 & 2.332457 & -0.012994 \\ \mathrm{C} & 0.000000 & 3.313572 & 1.551163 \\ \mathrm{P} & 0.000000 & -2.332457 & -0.012994 \\ \mathrm{C} & 0.000000 & -3.313572 & 1.551163 \\ \mathrm{C} & 1.716339 & 0.000000 & 0.946245 \\ \mathrm{O} & 2.650410 & 0.000000 & 1.663977 \\ \mathrm{C} & -1.716339 & 0.000000 & 0.946245 \\ & & & \\ & & & \mathrm{~S} 14 \\ \mathrm{O} & -2.650410 & 0.000000 & 1.663977 \\ \mathrm{C} & -1.433521 & -3.075092 & -0.915327 \\ \mathrm{C} & 1.433521 & -3.075092 & -0.915327 \\ \mathrm{C} & -1.433521 & 3.075092 & -0.915327 \\ \mathrm{C} & 1.433521 & 3.075092 & -0.915327 \\ \mathrm{H} & 0.000000 & 4.392933 & 1.340363 \\ \mathrm{H} & -0.889520 & 3.053766 & 2.140172 \\ \mathrm{H} & 0.889520 & 3.053766 & 2.140172 \\ \mathrm{H} & -1.336007 & 4.168377 & -0.985472 \\ \mathrm{H} & -1.491144 & 2.651333 & -1.927026 \\ \mathrm{H} & -2.361170 & 2.822940 & -0.384948\end{array}$




$\begin{array}{lrrr}\mathrm{H} & 1.336007 & 4.168377 & -0.985472 \\ \mathrm{H} & 2.361170 & 2.822940 & -0.384948 \\ \mathrm{H} & 1.491144 & 2.651333 & -1.927026 \\ \mathrm{H} & -1.336007 & -4.168377 & -0.985472 \\ \mathrm{H} & -2.361170 & -2.822940 & -0.384948 \\ \mathrm{H} & -1.491144 & -2.651333 & -1.927026 \\ \mathrm{H} & 1.336007 & -4.168377 & -0.985472 \\ \mathrm{H} & 1.491144 & -2.651333 & -1.927026 \\ \mathrm{H} & 2.361170 & -2.822940 & -0.384948 \\ \mathrm{H} & 0.000000 & -4.392933 & 1.340363 \\ \mathrm{H} & 0.889520 & -3.053766 & 2.140172 \\ \mathrm{H} & -0.889520 & -3.053766 & 2.140172 \\ - & \end{array}$

\section{Ru T :}

$\begin{array}{lrrr}\mathrm{E}(\mathrm{a} . \mathrm{u} .)=-6.23261 & & \\ \mathrm{Ru} & 0.000000 & 0.000000 & 0.2878861 \\ \mathrm{P} & 0.000000 & 2.304126 & -0.257523 \\ \mathrm{C} & 0.000000 & 3.460338 & 1.185404 \\ \mathrm{P} & 0.000000 & -2.304126 & -0.257523 \\ \mathrm{C} & 0.000000 & -3.460338 & 1.185404 \\ \mathrm{C} & 1.418524 & 0.000000 & 1.517860 \\ \mathrm{O} & 2.265623 & 0.000000 & 2.328256 \\ \mathrm{C} & -1.418524 & 0.000000 & 1.517860 \\ \mathrm{O} & -2.265623 & 0.000000 & 2.328256 \\ \mathrm{C} & -1.431417 & -2.966196 & -1.230068 \\ \mathrm{C} & 1.431417 & -2.966196 & -1.230068 \\ \mathrm{C} & -1.431417 & 2.966196 & -1.230068 \\ \mathrm{C} & 1.431417 & 2.966196 & -1.230068 \\ \mathrm{H} & 0.000000 & 4.510147 & 0.857223 \\ \mathrm{H} & -0.888786 & 3.268811 & 1.801210 \\ \mathrm{H} & 0.888786 & 3.268811 & 1.801210 \\ \mathrm{H} & -1.340679 & 4.053301 & -1.376087 \\ \mathrm{H} & -1.472755 & 2.469391 & -2.208429 \\ \mathrm{H} & -2.364331 & 2.748628 & -0.693384\end{array}$

$\begin{array}{llll}\mathrm{H} & -2.364331 & 2.748628 & -0.693384\end{array}$

\section{$\mathrm{S} 15$}

\begin{tabular}{|c|c|c|c|}
\hline $\mathrm{H}$ & 1.340679 & 4.053301 & -1.376087 \\
\hline $\mathrm{H}$ & 2.364331 & 2.748628 & -0.693384 \\
\hline $\mathrm{H}$ & 1.472755 & 2.469391 & -2.208429 \\
\hline $\mathrm{H}$ & -1.340679 & -4.053301 & -1.376087 \\
\hline $\mathrm{H}$ & -2.364331 & -2.748628 & -0.693384 \\
\hline $\mathrm{H}$ & -1.472755 & -2.469391 & -2.208429 \\
\hline $\mathrm{H}$ & 1.340679 & -4.053301 & -1.376087 \\
\hline $\mathrm{H}$ & 1.472755 & -2.469391 & -2.208429 \\
\hline $\mathrm{H}$ & 2.364331 & -2.748628 & -0.693384 \\
\hline $\mathrm{H}$ & 0.000000 & -4.510147 & 0.857223 \\
\hline $\mathrm{H}$ & 0.888786 & -3.268811 & 1.801210 \\
\hline $\mathrm{H}$ & -0.888786 & -3.268811 & 1.801210 \\
\hline
\end{tabular}

\section{Fe S :}

$\begin{array}{lrrr}\mathrm{E}(\mathrm{a} . \mathrm{u} .)=-2 & .59173 & & \\ \mathrm{Fe} & 0.000000 & 0.000000 & 0.405020 \\ \mathrm{C} & -1.605357 & 0.000000 & 1.149498 \\ \mathrm{C} & 1.605357 & 0.000000 & 1.149498 \\ \mathrm{C} & 0.000000 & 1.775280 & 0.088739\end{array}$




\begin{tabular}{|c|c|c|c|}
\hline $\mathrm{C}$ & 0.000000 & -1.775280 & 0.088739 \\
\hline 0 & 0.000000 & -2.883468 & -0.230908 \\
\hline 0 & -2.566276 & 0.000000 & 1.797270 \\
\hline 0 & 2.566276 & 0.000000 & 1.797270 \\
\hline O & 0.000000 & 2.883468 & -0.230908 \\
\hline
\end{tabular}

9Fe_T:

$\begin{array}{lrrr}\mathrm{E}(\mathrm{a} . \mathrm{u} .)=-2.59264 & & \\ \mathrm{Fe} & 0.000000 & 0.000000 & 0.270844 \\ \mathrm{C} & -1.351365 & 0.000000 & 1.461454 \\ \mathrm{C} & 1.351365 & 0.000000 & 1.461454 \\ \mathrm{C} & 0.000000 & 1.776553 & -0.204138 \\ \mathrm{C} & 0.000000 & -1.776553 & -0.204138 \\ \mathrm{O} & 0.000000 & -2.902346 & -0.451541 \\ \mathrm{O} & -2.205860 & 0.000000 & 2.238199 \\ \mathrm{O} & 2.205860 & 0.000000 & 2.238199 \\ \mathrm{O} & 0.000000 & 2.902346 & -0.451541\end{array}$

\section{Ru_S:}

$\begin{array}{lrrr}\mathrm{E}(\mathrm{a} . \mathrm{u} .)=-2 & .59730 & & \\ \mathrm{Ru} & 0.000000 & 0.000000 & 0.467077 \\ \mathrm{C} & -1.847346 & 0.000000 & 1.017790\end{array}$

\begin{tabular}{lrrr} 
& & & \multicolumn{1}{c}{ S16 } \\
$\mathrm{C}$ & 1.847346 & 0.000000 & 1.017790 \\
$\mathrm{C}$ & 0.000000 & 1.859063 & -0.051983 \\
$\mathrm{C}$ & 0.000000 & -1.859063 & -0.051983 \\
$\mathrm{O}$ & 0.000000 & -2.900157 & -0.549962 \\
$\mathrm{O}$ & -2.876553 & 0.000000 & 1.541014 \\
$\mathrm{O}$ & 2.876553 & 0.000000 & 1.541014 \\
$\mathrm{O}$ & 0.000000 & 2.900157 & -0.549962
\end{tabular}

9Ru_T:

\begin{tabular}{lrrr}
\hline E $($ a.u. $)=-2.57142$ & & \\
$\mathrm{Ru}$ & -0.184618 & -0.126546 & 0.000000 \\
$\mathrm{C}$ & -0.417758 & -0.474279 & 1.922979 \\
$\mathrm{C}$ & 1.701053 & 0.137806 & 0.000000 \\
$\mathrm{C}$ & -0.656550 & 1.718128 & 0.000000 \\
$\mathrm{C}$ & -0.417758 & -0.474279 & -1.922979 \\
$\mathrm{O}$ & -0.514842 & -0.618957 & 3.064597 \\
$\mathrm{O}$ & 2.837040 & 0.348450 & 0.000000 \\
$\mathrm{O}$ & -0.893335 & 2.848948 & 0.000000 \\
$\mathrm{O}$ & -0.514842 & -0.618957 & -3.064597
\end{tabular}

\section{CO :}

$\begin{array}{llll}\mathrm{E}(\mathrm{a} . \mathrm{u} .)=-0.54477 & & \\ \mathrm{C} & 0.000000 & 0.000000 & 0.208074 \\ \mathrm{O} & 0.000000 & 0.000000 & 1.343895 \\ & & \end{array}$


